\title{
Effects of fundamental structure parameters on dynamic responses of submerged floating tunnel under hydrodynamic loads
}

\author{
Xu Long • Fei Ge • Lei Wang • Youshi Hong
}

Received: 23 September 2008 / Revised: 2 December 2008 / Accepted: 11 December 2008

(C) The Chinese Society of Theoretical and Applied Mechanics and Springer-Verlag GmbH 2009

\begin{abstract}
This paper investigates the effects of structure parameters on dynamic responses of submerged floating tunnel (SFT) under hydrodynamic loads. The structure parameters includes buoyancy-weight ratio (BWR), stiffness coefficients of the cable systems, tunnel net buoyancy and tunnel length. First, the importance of structural damp in relation to the dynamic responses of SFT is demonstrated and the mechanism of structural damp effect is discussed. Thereafter, the fundamental structure parameters are investigated through the analysis of SFT dynamic responses under hydrodynamic loads. The results indicate that the BWR of SFT is a key structure parameter. When BWR is 1.2 , there is a remarkable trend change in the vertical dynamic response of SFT under hydrodynamic loads. The results also indicate that the ratio of the tunnel net buoyancy to the cable stiffness coefficient is not a characteristic factor affecting the dynamic responses of SFT under hydrodynamic loads.
\end{abstract}

Keywords Submerged floating tunnel - Structural damp · Buoyancy-weight ratio - Cable stiffness coefficient .

Tunnel net buoyancy $\cdot$ Hydrodynamic load

\section{Introduction}

Submerged floating tunnel (SFT), also named Archimedes Bridge, is a novel type of traffic structures for water crossings,

The project supported by the National Natural Science Foundation of China (10532070) and Chinese Academy of Sciences (KJCX2-YW-L07).

X. Long $\cdot$ F. Ge $\cdot$ L. Wang $\cdot$ Y. Hong $(\bowtie)$

State Key Laboratory of Nonlinear Mechanics,

Institute of Mechanics, Chinese Academy of Sciences,

Beijing 100190, China

e-mail: hongys@imech.ac.cn which is balanced by its buoyancy, self-weight and constraint forces resulted from cable systems and thus submerged a certain depth underwater. An SFT basically consists of four parts: (i) the tunnel structure which is made up of tunnel segments and allows traffics and pedestrians to get through, (ii) the shore connection structures which connect SFT to shores, (iii) the cable systems which are anchored to the waterbed to balance the net buoyancy (the present paper concentrates on the SFT type of tunnel buoyancy larger than tunnel weight), and (iv) the foundation structures which are constructed at the waterbed to install cable systems. One of the attractive advantages of SFT is that the construction cost of unit tunnel segment will not increase as the total length of SFT increases. Thus, SFT is more beneficial to be set up across the long-span water sound compared with traditional bridge structures. In addition, SFT is a preferred solution for the water area where traditional bridge construction is not suitable, due to the environmental-friendly features of SFT, i.e. less impact on surrounding natural environments and relatively less effect from typhoon and earthquake [1,2].

However, there is still not an actual SFT being built in the world. To build such a bridge will encounter various scientific and technical difficulties, such as the tunnel architecture design, the cable system configuration, the connection design between tunnel tube and shores, the installation of SFT structures, etc. Although a limited number of investigations may bring us useful premises and suggestions for the structure parameters and the design standard of construction, some essential technical issues related to safety, stability and reliability of SFT are still in the way of study, when it is subjected to hydrodynamic loads, seismic load and accidental load [3-5].

Compared with the case of traditional bridge structures, the research on dynamic responses of SFT under hydrodynamic loads is inevitable. Since 1980s, there have been 
research reports in the aspects of conceptual design and dynamic response by investigators from Italy, Norway, Japan, United States of America, etc. In China, the involvement of SFT study has started since 1990s. In recent years, the Sino-Italian Joint Laboratory of Archimedes Bridge (SIJ$\mathrm{LAB}$ ) has made efforts in the structural design of the first submerged floating tunnel prototype in Qiandao Lake of Zhejiang Province and has performed relevant simulations and experiments [6,7]. Nevertheless, as for such a novel structure, the mechanism of dynamic response under environmental loads is still not clear enough for the design of an SFT at present.

It is obvious that the structure design and the dynamic response study of SFT are noticeably dependent on hydrodynamic conditions (water wave and current [8]) where SFT is supposed to be constructed, and thus design parameters of the tunnel are related to the environment.

In the present paper, the effects of structure parameters on dynamic responses of SFT are investigated based on the hydrodynamic environment of Qiandao Lake and the structural design of the tunnel and cable system proposed by SIJLAB. The effect of structural damp on dynamic responses of SFT under hydrodynamic loads is discussed. Since dynamic responses in the current direction and in the vertical direction are the most dominating and meaningful in SFT design, the structure parameters related to the dynamic responses in both directions, such as buoyancy-weight ratio, cable stiffness coefficients, tunnel net buoyancy and tunnel length are analyzed.

\section{Calculation model}

In the calculation model of SFT, the structural design of the tunnel segment adopts the design scheme of submerged floating tunnel prototype (SFTP) in Qiandao Lake of Zhejiang Province. The design length of SFTP is $100 \mathrm{~m}$ and the tunnel is submerged $4.2 \mathrm{~m}$ under the water surface. The shore connections between SFTP and shores are of pin joints, while axial displacement relaxation is applied at one end. The configuration and the distribution of cable systems are schematically shown in Fig. 1, for which the tunnel tube is anchored by three cable groups to the foundation with the connection being of spherical hinges. Note that cable group 2 is located in the mid-span of SFTP and the other two groups (all named group 1) are symmetrically located at both sides of group 2. The parameters of SFTP structure and hydrodynamic environment of Qiandao Lake are listed in Table 1, which are used in the calculation.

The design of tunnel cross-section [7] is shown in Fig. 2, which is a sandwich structure consisting of outer aluminum shell, inner steel shell and concrete shell in the middle, for the aims of corrosion resistance, collision protection, tunnel weight balance, etc. A simplification method of total SFT cross-section based on the principle of bending stiffness equivalence [5] is employed in the calculation. That is

$E I=E_{A} I_{A}+E_{C} I_{C}+E_{S} I_{S}$,

where $E_{A}, E_{C}$ and $E_{S}$ are the elastic moduli of aluminum, concrete and steel, respectively; $I_{A}, I_{C}$ and $I_{S}$ are the crosssectional moments of inertia of aluminum extrusion, concrete shell and steel shell, respectively; $E$ is the equivalent elastic modulus of the simplified tunnel; and $I$ is the total cross-sectional moments of inertia of the simplified tunnel. With the same diameter of tunnel and the same mass of unit length along the tunnel, the equivalent elastic modulus of the tunnel in the form of the proposed sandwich shell can be obtained.

Morison Equation expressed by Eq. (2) and Stokes fifth order wave theory are employed to calculate fluid forces of the tunnel and the cables subjected to hydrodynamic loads [9].

$$
\begin{aligned}
f(t)= & \frac{1}{2} C_{D} \rho D\left(u_{w}+u_{c}-\dot{x}_{i}\right)\left|u_{w}+u_{c}-\dot{x}_{i}\right| \\
& +\frac{\rho \pi D^{2}}{4}\left(C_{m} \dot{u}_{w}-C_{a} \ddot{x}_{i}\right), \quad(i=1,2),
\end{aligned}
$$

where $x_{i}(i=1,2)$ is the displacement in $X$ or $Z$ direction; $u_{w}$ and $u_{c}$ are the fluid particle velocities on the axis of SFTP in $X$ or $Z$ direction; and other parameters are defined in Table 1. a

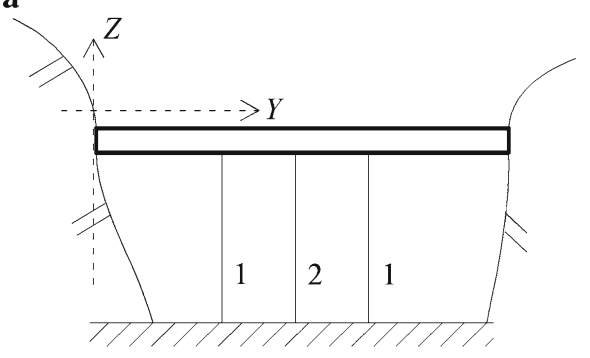

b

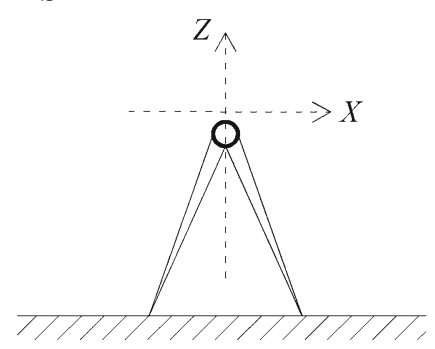

c

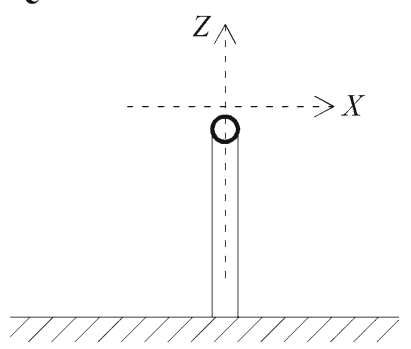

Fig. 1 Schematic diagram of SFTP, tunnel tube anchored by three cable groups to foundation. a Side elevation; b Cable group 2; c Cable group 1 
Table 1 Parameters of fluid dynamic environment and SFTP structure

\begin{tabular}{llllllcc}
\hline Fluid dynamic environment & Symbol & Unit & Value & Structural properties & Symbol & Unit & Value \\
\hline Fluid density & $\rho$ & $\mathrm{kg} / \mathrm{m}^{3}$ & 1,050 & Tunnel equivalent density & $\rho_{\mathrm{T}}$ & $\mathrm{kg} / \mathrm{m}^{3}$ & 2,018 \\
Water depth & $h$ & $\mathrm{~m}$ & 30 & Tunnel outer diameter & $D$ & $\mathrm{~m}$ & 4.39 \\
Wave height & $H$ & $\mathrm{~m}$ & 1.0 & Tunnel inner diameter & $d$ & $\mathrm{~m}$ & 3.48 \\
Wave period & $T$ & $\mathrm{~s}$ & 1.8 & Tunnel equivalent Young modulus & $E_{\mathrm{T}}$ & $\mathrm{N} / \mathrm{m}^{2}$ & $3.2 \times 10^{10}$ \\
Surface current velocity & $U_{0}$ & $\mathrm{~m} / \mathrm{s}$ & 0.1 & Cable density & $\rho_{\mathrm{C}}$ & $\mathrm{kg} / \mathrm{m}^{3}$ & 7,850 \\
Drag coefficient & $C_{\mathrm{D}}$ & 1 & 1.0 & Cable diameter & $d_{\mathrm{C}}$ & $\mathrm{m}$ & 0.06 \\
Mass coefficient & $C_{\mathrm{m}}$ & 1 & 2.0 & Cable Young modulus & $E_{\mathrm{C}}$ & $\mathrm{N} / \mathrm{m}^{2}$ & $1.4 \times 10^{11}$ \\
Added-mass coefficient & $C_{\mathrm{a}}$ & 1 & 1.0 & Kinetic viscosity Coefficient & $v$ & $\mathrm{~m}^{2} / \mathrm{s}$ & $1.067 \times 10^{-6}$ \\
\hline
\end{tabular}

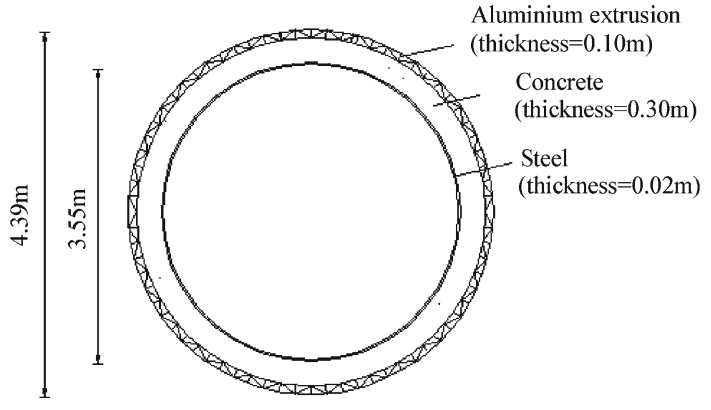

Fig. 2 Designed cross section of SFTP [7]

During the calculation, energy loss due to the deformation and the movement of the tunnel structure notably influences the dynamic responses of SFTP and the effect of energy loss in the form of structure damp cannot be neglected. However, for the structural damp of actual structures is hardly to be provided precisely, it is usually obtained by means of practical measurements of specific structures. Thus, Rayleigh damping model is adopted in the calculation. That is, the damping matrix of the physical system having multi-degrees of freedom can be treated as the sum of the mass matrix and the stiffness matrix for the system taking the form of $[C]=\alpha[M]+\beta[K]$, with the damping coefficients $\alpha$ and $\beta$ being

$\alpha=\frac{2 \xi_{i} \omega_{i} \omega_{j}^{2}-2 \xi_{j} \omega_{j} \omega_{i}^{2}}{\omega_{j}^{2}-\omega_{i}^{2}}, \quad \beta=\frac{2 \xi_{j} \omega_{j}-2 \xi_{i} \omega_{i}}{\omega_{j}^{2}-\omega_{i}^{2}}$,

where $\omega_{i}$ and $\omega_{j}$ are the $i$ th order and the $j$ th order natural frequencies of structure, respectively; and $\xi_{i}$ and $\xi_{j}$ are the $i$ th order and the $j$ th order damping ratios of structure, respectively.

With all of the considerations regarding structure configuration, loading calculation and damping effect, the transient solver of ANSYS and the pipe59 element are applied to simulate SFT subjected to gravity, buoyancy, cable tensions and fluid forces.

\section{Effect of structural damp on SFT}

The modal analysis method in a small damping system is used to solve the natural frequencies of SFT. Owing to the coupling between tunnel and cable systems, low order frequencies and modal shapes of SFT mainly result from cable systems, when the cable systems have been meshed into pipe59 elements. The commonly used solution scheme in cable-stayed bridge analysis is also brought into the present calculation model. That is, each cable in the calculation model is meshed with a single pipe59 cable element for the modal analysis, and the first two order frequencies and modal shapes of SFT dominated by tunnel vibration can be obtained. The modal analysis results regarding SFTP, as a simulation example, are shown in Fig. 3. The natural frequencies corresponding to the first and the second modal shapes of SFTP are 0.815 and $1.45 \mathrm{~Hz}$.

The damping ratio of each order mode from real SFT is unavailable. But it is noted that the damping ratios of the first and the second order are almost the same while the first order is the major excited one in the present case. Thus, in the calculation, the damping ratio of 0.025 measured in model experiments of SFTP [10] is used for the first and the second order mode. The damping coefficients $\alpha$ and $\beta$ can be calculated with Eq. (3) and the results are 0.586 and 0.00352 , respectively. Thus, dynamic responses of SFTP including structural damping can be analyzed numerically. For both cases of with and without structural damping effect, the dynamic responses at SFTP mid-span in current direction and vertical direction are shown in Fig. 4.

Figure 4 shows that in the current direction the amplitudes of both cases are almost equal to each other, although a phase shift exists; while in the vertical direction the differences of the amplitudes and the frequencies of dynamic responses with and without structural damp are remarkable. Therefore, the effect of structural damp on dynamic response is evident in the vertical direction, whereas the effect is negligible in the current direction.

The data shown in Fig. 4 are the simulation results for the time scale between 40 and $50 \mathrm{~s}$, which are of the steady state 
Fig. 3 The first and the second order modal shapes of SFTP. a The first order modal shape; b The second order modal shape

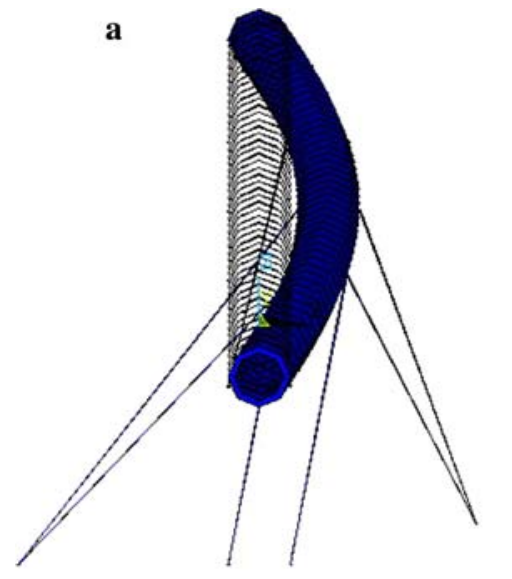

b
Fig. 4 Dynamic responses at the mid-span of SFTP under hydrodynamic loads versus time. a Current direction; b Vertical direction
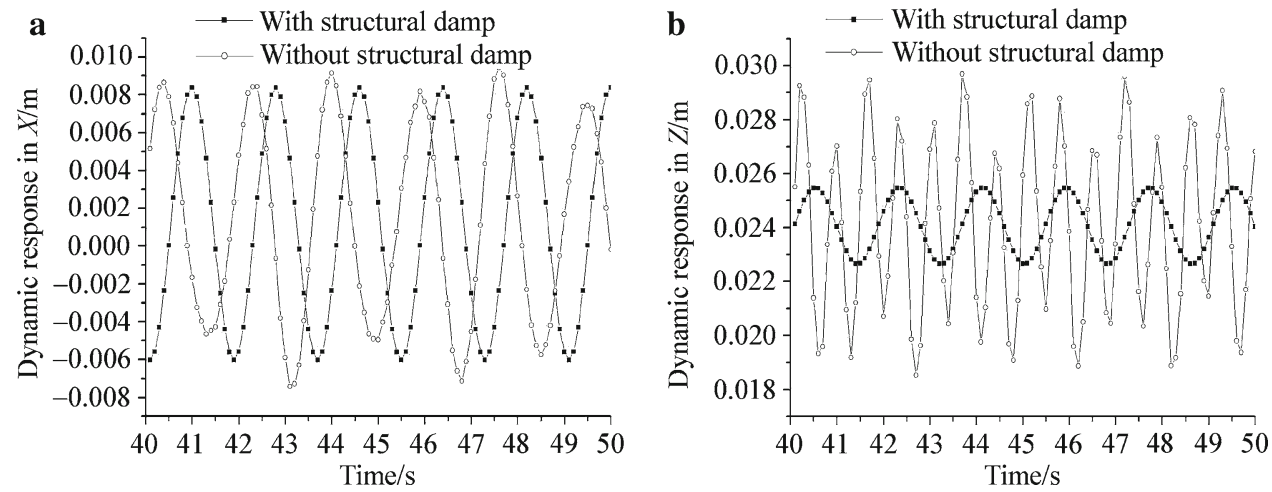

of dynamic responses for SFTP under hydrodynamic loads. It implies that in the case of structural damp neglected, low order vibration modals of the tunnel are excited and the vertical dynamic response of SFTP is the superposition of the first order modal vibration to the forced vibration under hydrodynamic loads. On the other hand, in the case of the structural damp considered, the vertical dynamic response of SFTP is only the forced vibration of the tunnel under hydrodynamic loads, while the low order modals are not excited.

Furthermore, for the case of structural damp neglected, the dynamic responses of SFTP under hydrodynamic loads with different BWRs are analyzed. The relationship between dynamic responses at SFTP mid-span in the current and vertical directions and BWR are shown in Fig. 5, where the ordinate is the standard deviation (STDEV) of the dynamic response in certain direction normalized by the tunnel diameter (D), while the abscissa is the buoyancy-weight ratio (BWR). Similarly, for the case of structural damp considered, the relationships between STDEV of SFTP dynamic responses and BWR are shown in Fig. 6. Here, STDEV is defined as the small amplitude vibration of tunnel around its updated balance location induced by hydrodynamic loads, i.e. the value of STDEV is the real reflection of dynamic response of tunnel tube under hydrodynamic loads.
The comparison of Figs. 5b-6b indicates that structural damp is not a negligible factor in the analysis of dynamic responses of SFT under hydrodynamic loads. Figure 6 demonstrates that dynamic responses in both current and vertical directions decrease as BWR increases from 1.1 to 1.9. When BWR is larger than 1.2, STDEV of dynamic response in the vertical direction is, to some degree, of the steady state as shown in Fig. 6b. That is, even if BWR increases from 1.2 to 1.9 , the decrease of the STDEV value of dynamic response in the vertical direction is less evident.

From the discussion regarding dynamic responses based on the assumption of Rayleigh damping model, it is noticed that structural damp substantially influences the calculation results of dynamic response analysis involving SFTP with different BWRs under hydrodynamic loads. When the structural damp is taken into consideration, the dynamic responses of SFTP decrease and the influence pattern of different BWRs on dynamic responses is also changed. It is also noted that damping coefficient $\alpha$ increases while $\beta$ decreases as BWR increases from 1.1 to 1.9 by comparing damping coefficients $\alpha$ and $\beta$ in SFTPs with different BWRs. In other words, in such a circumstance, the influence of the mass matrix on structural damp increases, while the influence of the stiffness matrix on structural damp decreases. 
Fig. 5 Relationship between STDEV of SFTP dynamic responses and BWR (without structural damp). a Current direction; b Vertical direction

Fig. 6 Relationship between STDEV of SFTP dynamic responses and BWR (with structural damp). a Current direction; b Vertical direction
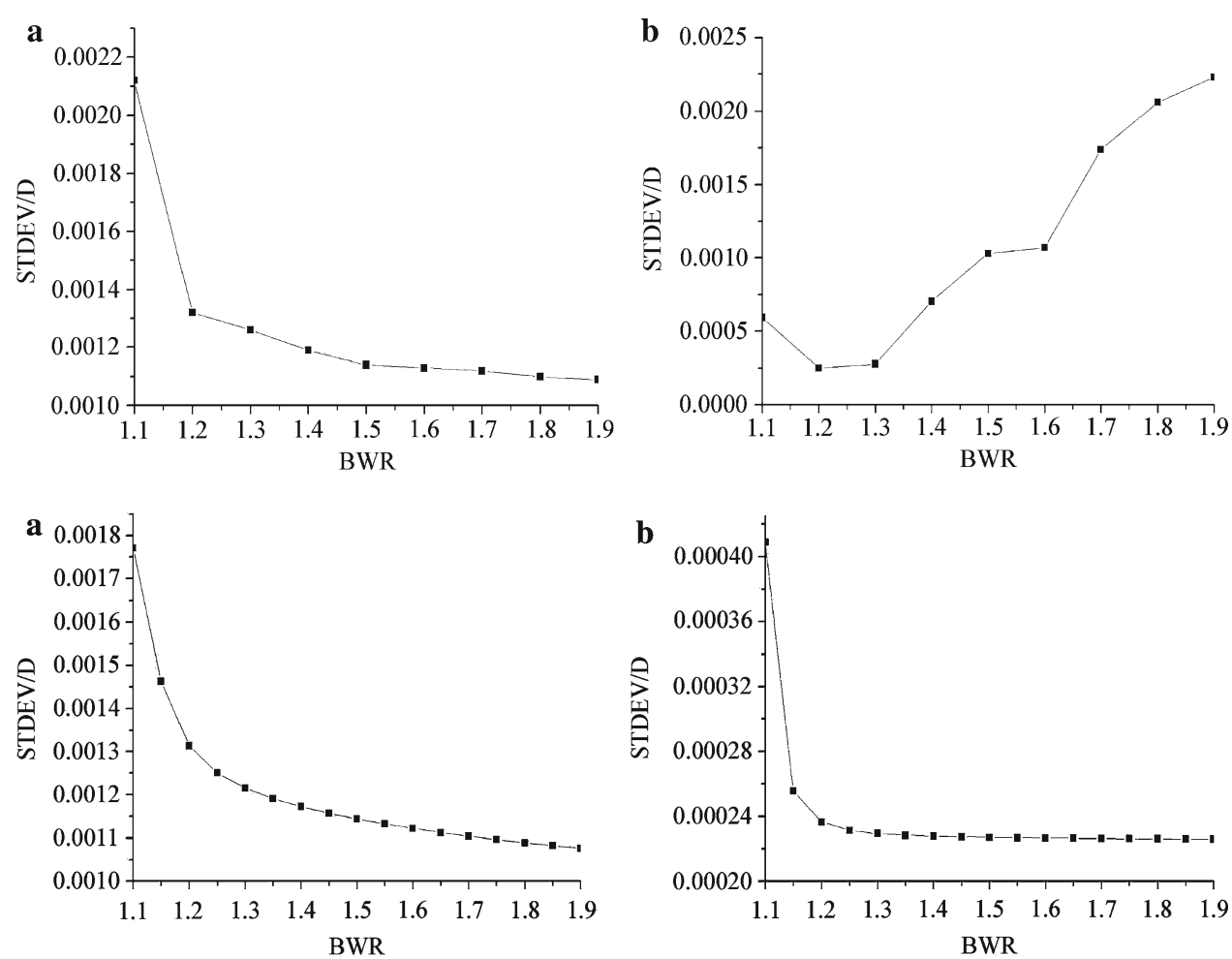

\section{Effects of fundamental structure parameters on dynamic responses of SFTP}

\subsection{Tunnel length}

The tunnel length is one of the fundamental structure parameters in the engineering design of SFTP, which is obviously determined by the distance of water crossing and will definitely affect the compatibility condition of tunnel deformation, the configuration of cable systems, the net buoyancy of tunnel tube and concentrated forces at the shore connections. If the SFTP structure is assumed as a unit tunnel segment, then a long SFT can be built by coupling all of the degrees of freedom of adjacent segment ends. For example, a long SFT structure formed by five tunnel segments is shown in Fig. 7 and the first two order natural frequencies and modal shapes due to tunnel vibration are shown in Fig. 8.

In order to discuss the effect of tunnel length on the dynamic responses of SFT under hydrodynamic loads, ten cases with different tunnel lengths $(100-1,000 \mathrm{~m})$ are calculated by coupling different numbers of tunnel segments (the length of unit tunnel segment is $100 \mathrm{~m}$ ), in which the hydrodynamic conditions in Qiandao Lake is used.

Dynamic responses in both current direction and vertical direction at the mid-span of SFTs with different tunnel lengths are shown in Fig. 9, where the ordinate is the ratio of dynamic response STDEV to tunnel diameter (D), while the abscissa is the ratio of tunnel length $(\mathrm{L})$ to tunnel diameter.

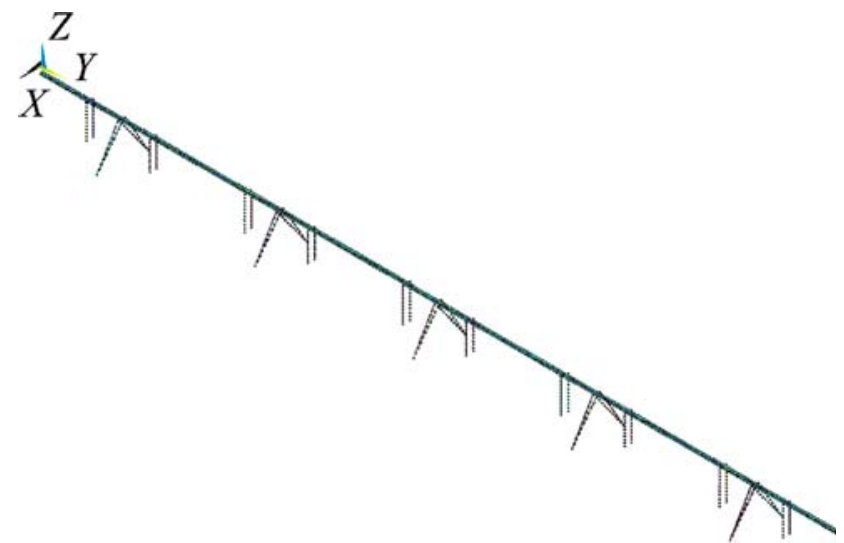

Fig. 7 Schematic diagram of an SFT formed by five tunnel segments
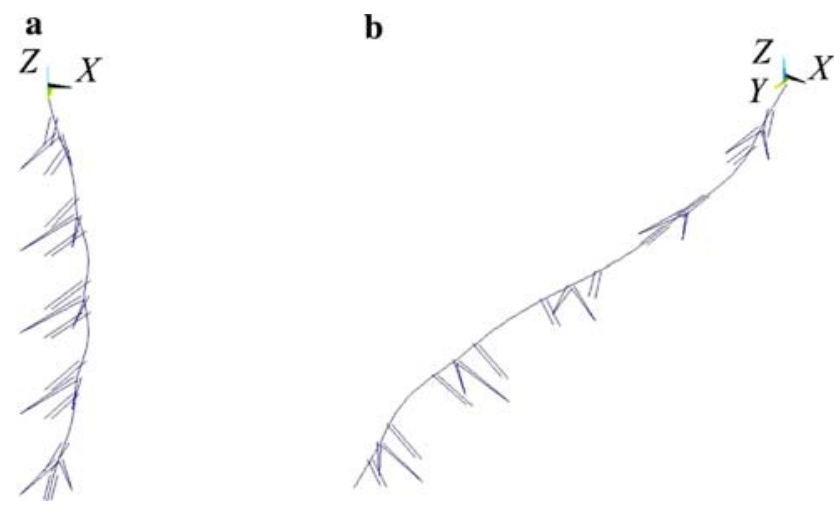

Fig. 8 The first and the second order modal shapes of an SFT. a The first order modal shape; $\mathbf{b}$ The second order modal shape 
Fig. 9 Relationship between tunnel length and STDEV of dynamic responses. a Current direction; b Vertical direction

Fig. 10 Dynamic responses at SFT mid-span with different BWRs versus time. a Current direction; b Vertical direction
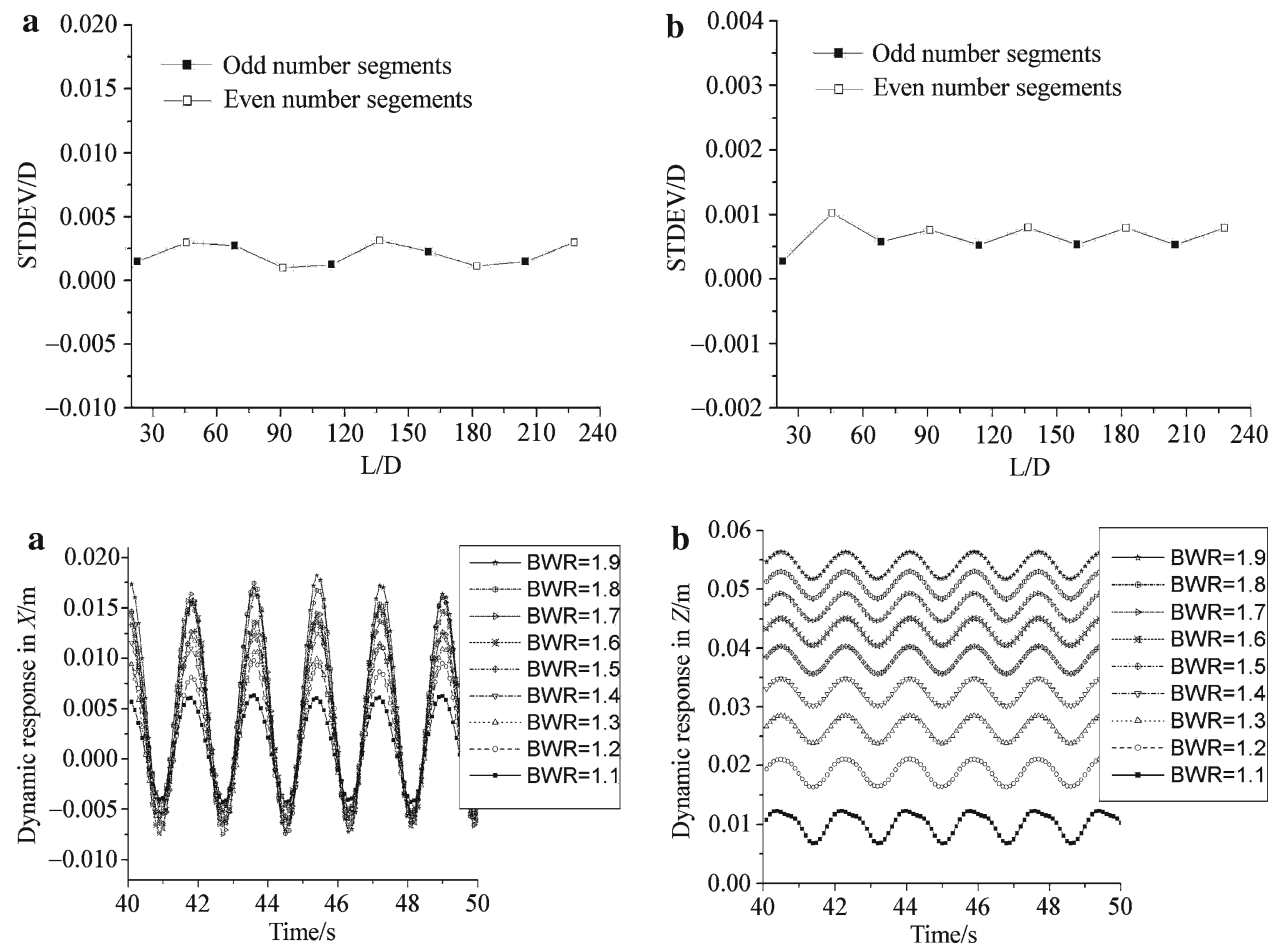

It is observed in Fig. 9a that although the value of STDEV in current direction exhibits a sine-shape pattern as the tunnel length increases, the value of STDEV is far less than that of tunnel diameter, which implies that the effect of the tunnel length on the dynamic response in the current direction is negligible.

In the vertical direction as shown in Fig. 9b, the regular fluctuation in the dynamic response curve mainly results from the difference of constraint strength. In the case of SFTs with odd number tunnel segments (the cases with tunnel lengths of $100,300,500 \mathrm{~m}$, etc.), the mid-span of SFT is just the constraint point of cable system and the constraint strength of mid-span is much stronger than the adjacent points, so the amplitude of the mid-span dynamic response is not the maximum point along the tunnel. In the case of SFTs with even number tunnel segments (the cases with tunnel lengths of $200,400,600 \mathrm{~m}$, etc.), the mid-span of SFTs is the connection point between tunnel segments and there is no external constraint, so the amplitude of mid-span dynamic response is the largest one along the tunnel. Therefore, the constraint strength of odd-number-segment SFT is stronger than that of even-number-segment SFT. That is, under the same applied loads, the amplitudes of the mid-span dynamic response of the odd-number-segment SFTs are less than those of the even-number-segment SFTs. Nevertheless, only small fluctuation of the STDEV curve prevails in the vertical direction, i.e. the dynamic response of SFT does not evidently affected by the tunnel length.

In brief, the effect of the tunnel length on the dynamic responses of SFT regarding the environmental background of Qiandao Lake exists but is not substantial. In the engineering design of SFTP, the effect of tunnel length on the amplitude of dynamic response is insignificant.

\subsection{Buoyancy-weight ratio}

The buoyancy-weight ratio (BWR) is defined as the ratio of tunnel buoyancy to the whole tunnel weight (including weights of tunnel structure, infrastructure, and cable systems). For such a structure, BWR influences not only the tunnel geometrical design but also material selection, strength design, stiffness design, etc. It is undoubted that tunnel buoyancy must be larger than its self-weight and the net buoyancy (the difference between buoyancy and self-weight) is balanced by the cable systems which are connected between tunnel and foundation. Obviously, BWR determines the tension force of the cable section and influences the dynamic behavior of SFT structure (tunnel and cables [11]), especially the stability of SFT. Furthermore, under the service condition, the actual BWR value of SFT is affected by traffic load and crowd load. So it is an important issue in the feasibility analysis to find out an adequate range of BWR for which the responses of SFT can show a steady performance.

Consider, for example, an SFT structure with five tunnel segments for simulation as shown in Fig. 7. After modal analysis and structural dynamic response analysis with structural damp, the displacement oscillations at the SFT midspan as a function of time history are obtained and shown in Fig. 10. Dynamic responses of SFTs with different BWRs in both current direction and vertical direction are shown in 
Fig. 11 Relationship between STDEV of dynamic responses at SFT mid-span and BWR. a Current direction; b Vertical direction

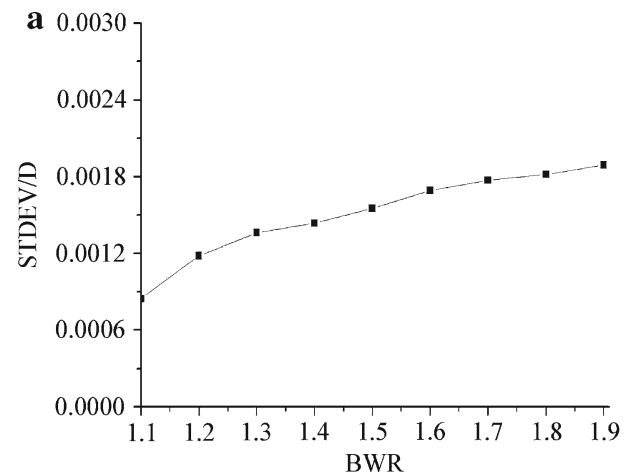

Fig. 11, where the ordinate is the ratio of STDEV of dynamic responses to the tunnel diameter, while the abscissa is BWR.

Figures 10 and 11 demonstrate the BWR effect on SFT dynamic responses due to hydrodynamic loads. As for the dynamic response in the current direction, the value of STDEV increases with the increasing value of BWR. As shown in Fig. 11a, when BWR is between 1.1 and 1.2, the value of STDEV in the vertical direction decreases steeply with the increase in BWR, comparing with the BWR larger than 1.2. As discussed in the previous part of "Effect of structural damp on SFT", the value of BWR may affect the value of damping coefficients ( $\alpha$ increases while $\beta$ decreases as BWR increases) in the assumption of Rayleigh damping model and, consequently, make a considerable impact on the value of the integral structural damp. Thus, the dynamic response of SFT under hydrodynamic loads versus BWR is probably due to the involvement of the structural damp.

From Rayleigh damping model, it is obvious that in addition to the influences of damping coefficients $\alpha$ and $\beta$ on the structural damp, the structural damp also depends on the relative value of the mass matrix and the stiffness matrix in every specific design. For instance, in a certain design, the contribution of the mass matrix is much less than that of the stiffness matrix of the structure. Then, as BWR increases, the decreasing value of $\beta$ is of a major advantage in the calculation of structural damp; while the increase of structural damp resulted from the increasing value of $\alpha$ only presents a negligible influence on the total value of structural damp. Therefore, it is the contribution extent from the mass matrix and the stiffness matrix that determines the response patterns due to different values of BWR, i.e. the increase of BWR can bring about the increase of dynamic responses of SFT in some designs and also may lead to the decrease of dynamic responses of SFT in other designs.

The present calculation results as well as our previous simulations [12] demonstrate that no matter whether the structural damp is included or not, when BWR of tunnel is larger than 1.2, there is an evident trend change in the dynamic response of the vertical direction of SFT under hydrodynamic loads.

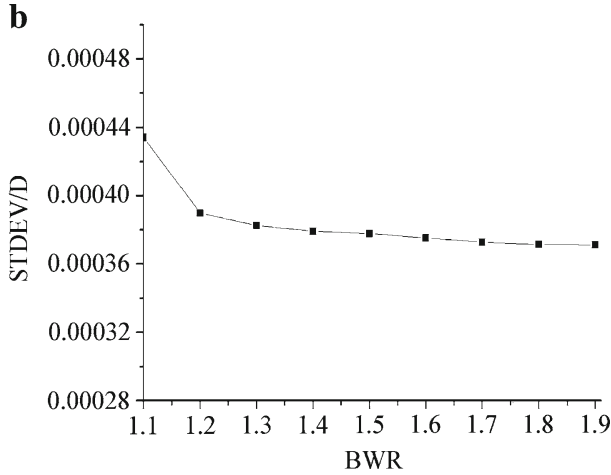

\subsection{Cable system stiffness}

As this paper only concerns the SFT type with BWR larger than unity, the tunnel buoyancy is larger than tunnel selfweight and the net buoyancy is balanced by cable systems which are assembled between tunnel and foundation. Hence, the design parameters of cable systems will directly determine relevant anchoring abilities and, consequently, influence dynamic responses of SFT under hydrodynamic loads. The SFT stiffness matrix is calculated synchronously with SFT deformation in the time history via ANSYS. In order to analyze the effect of cable system stiffness on the dynamic responses of SFT tunnel, the stiffness formulas of cable systems in $X$ and $Z$ directions are given in this part to deal with the results from ANSYS postprocessors. With regard to the ratio of the net buoyancy of unit tunnel segment $(100 \mathrm{~m})$ to the stiffness coefficient of the cable systems (BCR), the effect of the cable system stiffness on dynamic responses of SFT under hydrodynamic loads is discussed.

By concerning the constraint nonlinearity of cable systems, some calculation results both in the static and dynamic analyses showed that the deformation of cable system due to the gravity effect is negligible compared with the dynamic responses due to hydrodynamic loads [13]. Thus, elastic supports are employed to simulate the SFT cable systems in the analysis. The schematic diagram of stiffness calculation for cable group 1 is shown in Fig. 12 [10].

If the displacement of tunnel in $X$ direction is $\delta x$ as shown in Fig. 12a, then according to the geometric relationship and Hooke's Law, the change of tension $\left(\Delta T_{x}\right)$ in cable group 1 is derived as

$\Delta T_{x}=\frac{A E}{L}\left(\sqrt{\delta x^{2}+L^{2}}-L\right)$,

where $A, E$ and $L$ are sectional area, elastic modulus and initial length of each cable, respectively. The force equilibrium in $X$ direction leads to

$K_{x} \delta x=4\left(T_{0}+\Delta T_{x}\right) \sin \theta_{x}$, 
Fig. 12 Schematic diagram of cable group 1. a Surge motion; b Heave motion

Fig. 13 Schematic diagram of cable group 2. a Surge motion; b Heave motion a
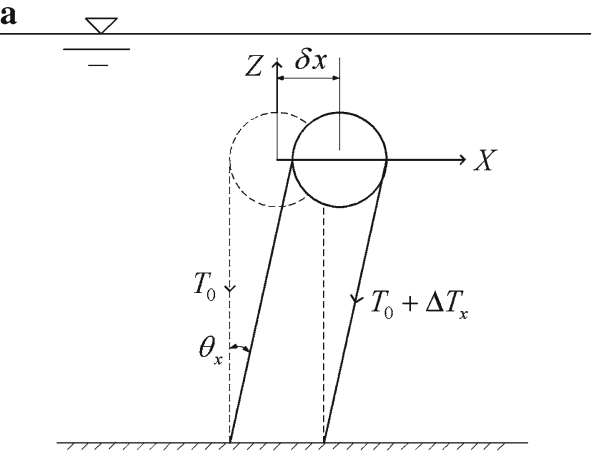

a

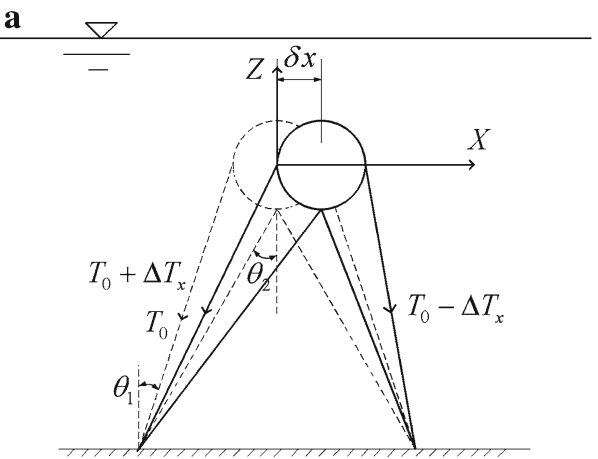

b

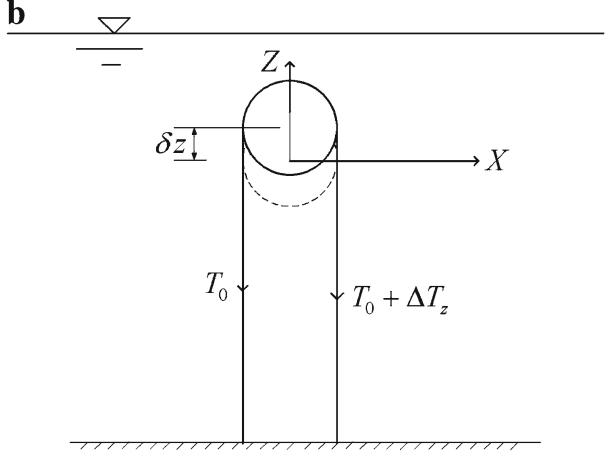

b

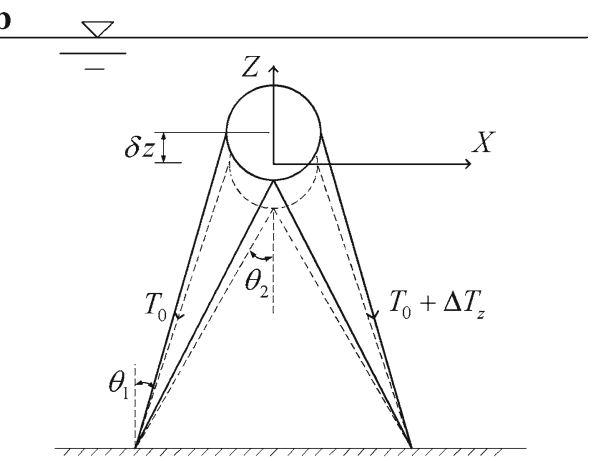

where $K_{x}$ is the stiffness of cable group 1 in $X$ direction, $T_{0}$ is the initial tension and $\theta_{x}$ is the inclined angle of each cable during the vibration process. According to the geometric relation, $\sin \theta_{x}=\delta x / \sqrt{\delta x^{2}+L^{2}}$ and $\cos \theta_{x}=L / \sqrt{\delta x^{2}+L^{2}}$, which are substituted into Eq. (5) to yield

$$
\begin{aligned}
K_{x} & =\frac{4\left(T_{0}+\Delta T_{x}\right)}{\sqrt{\delta x^{2}+L^{2}}} \\
& =\frac{4 T_{0}}{\sqrt{\delta x^{2}+L^{2}}}+\frac{4 E A}{L}\left(1-\frac{L}{\sqrt{\delta x^{2}+L^{2}}}\right) .
\end{aligned}
$$

If the displacement of the tunnel in $Z$ direction is $\delta z$ as shown in Fig. 12b, then the change of tension $\left(\Delta T_{z}\right)$ in cables is obtained as $\Delta T_{z}=E A \delta z / L$.

From the force equilibrium relation $K_{z} \delta z=4 \Delta T_{z}=$ $4 E A \delta z / L$, the stiffness of cable group 1 in $Z$ direction is derived as

$K_{z}=4 E A / L$

The schematic diagram of stiffness calculation of cable group 2 is shown in Fig. 13 [13]. Similarly, if the displacement of tunnel in $X$ direction is $\delta x$ and the change of length of outer cables of group 2 is $\Delta L_{\mathrm{O}}$ as shown in Fig. 13a, then the change of tension in cables is obtained as $\Delta T_{x}=A E \Delta L_{\mathrm{o}} / L$, where $A, E$ and $L$ are sectional area, elastic modulus and initial length of outer cable, respectively.

In accordance to the geometric relation, it is assumed that $\Delta L_{\mathrm{o}} / \delta x=\sin \theta_{1}$, then the force equilibrium equation in $X$ direction is

$K_{x \mathrm{o}} \delta x=\left(T_{0}+\Delta T_{x}\right) \sin \theta_{1}-\left(T_{0}-\Delta T_{x}\right) \sin \theta_{1}$,

where $T_{0}$ and $\theta_{1}$ are the initial tension and initial inclined angle of outer cables, respectively. Thus, Eq. (8) becomes $K_{x \mathrm{o}}=\left(2 E A \sin ^{2} \theta_{1}\right) / L_{1}$.

As shown in Fig. 13b, if the displacement of tunnel in $Z$ direction is $\delta z$ and the change of length of outer cables for group 2 is $\Delta L_{0}$, then the change of tension in cables is obtained as $\Delta T_{z}=A E \Delta L_{\mathrm{o}} / L_{1}$. Again referred to the geometric relation, it is assumed that $\Delta L_{\mathrm{o}} / \delta z=\cos \theta_{1}$, and the force equilibrium in $Z$ direction is

$K_{z o} \delta z=\Delta T_{z} \cos \theta_{1}+\Delta T_{z} \cos \theta_{1}$.

Thus, one obtains $K_{z o}=\left(2 E A \cos ^{2} \theta_{1}\right) / L_{1}$.

From the foregoing discussion, the formulas of stiffness coefficients of SFTP cable systems can be written as

$$
\begin{aligned}
K_{x}= & \frac{2 E A \sin ^{2} \theta_{1}}{L_{1}}+\frac{2 E A \sin ^{2} \theta_{2}}{L_{2}} \\
& +\frac{4 E A\left(1-L_{3} / \sqrt{\delta x^{2}+L_{3}^{2}}\right)}{L_{3}}+\frac{4 T_{0}}{\sqrt{\delta x^{2}+L_{3}^{2}}}, \\
K_{z}= & \frac{2 E A \cos ^{2} \theta_{1}}{L_{1}}+\frac{2 E A \cos ^{2} \theta_{2}}{L_{2}}+\frac{4 E A}{L_{3}},
\end{aligned}
$$

where $L_{1}$ and $\theta_{1}$ are the initial length and the inclined angle of outer cables of group $2, L_{2}$ and $\theta_{2}$ are the initial length and the inclined angle of inner cables of group $2, L_{3}$ is the initial 
Fig. 14 Relationship between STDEV and BCR. a Current direction; b Vertical direction

Fig. 15 Relationship between STDEV and BCR. a Current direction; b Vertical direction
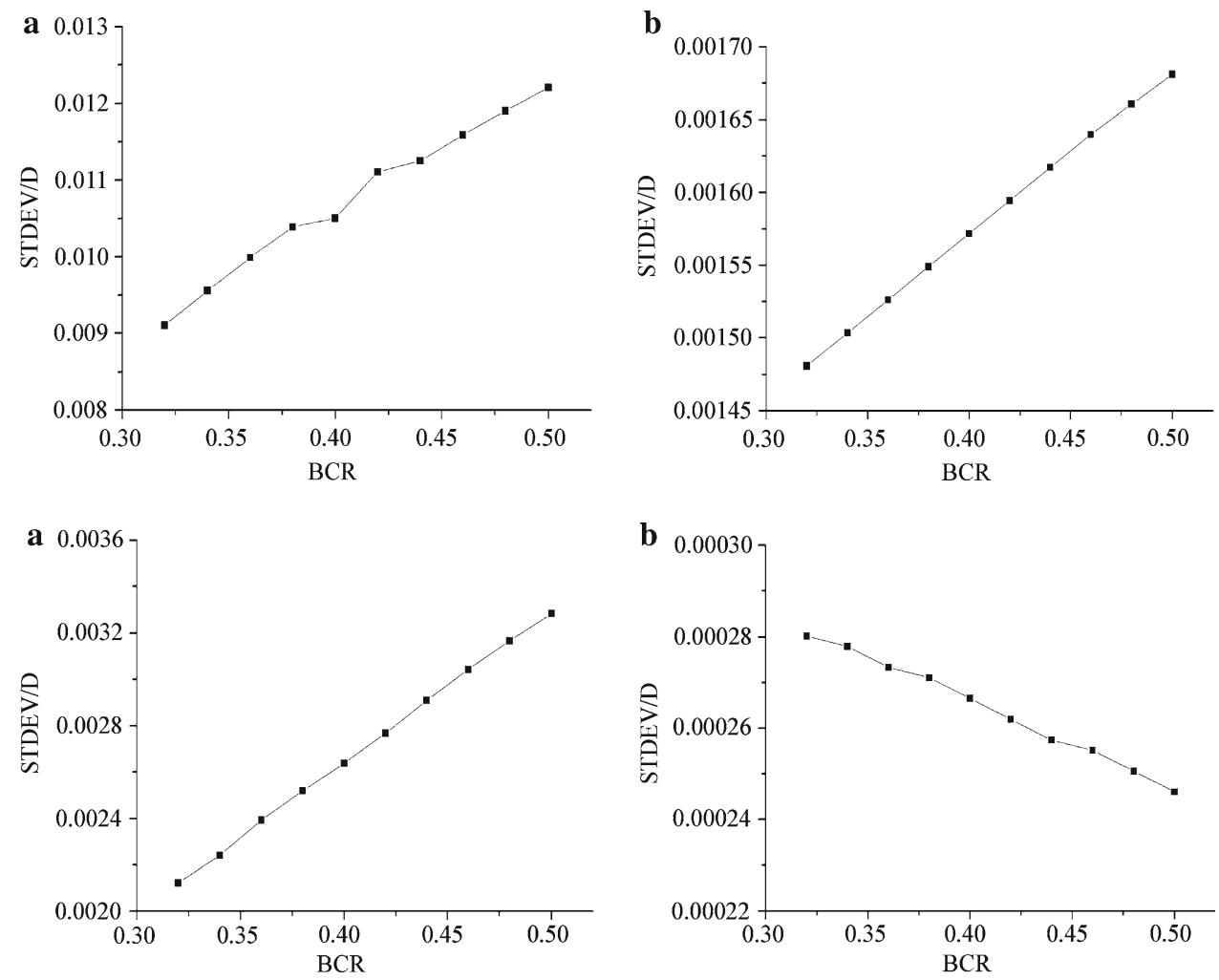

length of vertical cables of group 1 and $\delta x$ is the displacement of tunnel in the current direction.

By adjusting the elastic modulus of cable material in the simulation, the stiffness coefficients of both cable groups can be adjusted and the influence of BCR on dynamic responses can be analyzed. The BCR range between 0.32 and 0.50 is calculated and the results of STDEV for SFTP dynamic responses are shown in Fig. 14.

\subsection{Net buoyancy of unit tunnel segment}

Equation (11) indicates that vertical stiffness coefficient of cable systems is irrelevant with the initial tension of cables. Hence, the vertical stiffness coefficient of cable systems can be assumed as the reference of the net buoyancy change in the discussion of net buoyancy effect.

By adjusting the outer diameter of tunnel, the net buoyancy of unit tunnel segment can be adjusted and, consequently, the influence of BCR can be analyzed from the net-buoyancy point of view. The BCR range between 0.32 and 0.50 is calculated and the simulation results of STDEV for SFTP dynamic responses are shown in Fig. 15.

Figure 15 indicates that as for the structure parameters of the cable systems employed in this paper, the value of STDEV for SFTP dynamic response in the current direction increases as the net buoyancy of unit tunnel segment increases, while in the vertical direction STDEV of SFTP dynamic response increases as the net buoyancy of unit tunnel segment decreases. Moreover, the relationships between STDEV and BCR in both current direction and vertical direction show obvious linearity characteristics.

According to the comparison of Figs. $14 \mathrm{~b}$ and $15 \mathrm{~b}$, and the discussion in Sects. 4.3 and 4.4, it is reasonable to judge that on the premise of SFT structural safety (BCR is in the range between 0.32 and 0.50 ), $\mathrm{BCR}$ is dimensionless but not a characteristic factor with respect to SFT dynamic response under hydrodynamic loads. However, it is certain that both of the net buoyancy and the stiffness coefficient of cable systems are essential factors in the research and the design of SFT.

\section{Conclusions}

By the solution of SFT calculation model and the evaluation on fundamental structure parameters regarding the structure design of SFTP, the following conclusions are drawn:

(1) BWR is a key structure parameter regarding the SFT structural stability. The effect of BWR on SFT dynamic responses under hydrodynamic loads are affected by the contribution of the mass matrix and the stiffness matrix in a design. No matter whether structural damp is considered or not, when BWR is 1.2 , there is a remarkable trend change in the vertical dynamic response of SFT under hydrodynamic loads. 
(2) The net buoyancy of tunnel segment and the stiffness coefficient of cable systems are significant structure parameters making influence on SFT dynamic responses. However, BCR is not a characteristic factor with respect to SFT dynamic responses under hydrodynamic loads.

(3) Structural damp is an inevitable factor in the analysis of SFT dynamic responses under hydrodynamic loads. Structural damp evidently affects the amplitude and the frequency of dynamic response in the vertical direction, whereas in the current direction the structural damp effects on the dynamic response amplitude are negligible.

(4) The effect of SFT tunnel length on dynamic responses can be neglected in the practical design.

\section{References}

1. Ahrens, D.: Submerged floating tunnels: a concept whose time has arrived. Tunn. Undergr. Space Technol. 11, 505-510 (1996)

2. Huang, G.J., Wu, Y.X., Hong, Y.S.: Transportation of crossing waterways via Archimedes bridges. Ship Build. China 43 (supplement), 13-18 (2002) (in Chinese)

3. Mai, J.T., Yang, X.C., Guan, B.S.: Dynamic response analysis of the submerged floating tunnel subjected to wave. J. Railw. Eng. Soc. 102, 45-49 (2007) (in Chinese)

4. Fogazzi, P., Perotti, F.: The dynamic response of seabed anchored floating tunnels under seismic excitation. Earthq. Eng. Struct. Dyn. 29, 273-295 (2000)
5. Hui, L.: Dynamic response of submerged floating tunnel under accident loading. [M.S. Dissertation], Institute of Mechanics, Graduate University of Chinese Academy of Sciences, Beijing, China (2007) (in Chinese)

6. Chinese team of SIJLAB: Report of Research and Design for Archimedes Bridge Prototype at Qiandao Lake. Beijing, China, SIJLAB (2007)

7. Italian team of SIJLAB: Design Report of the Archimede's Bridge Prototype in Qiandao Lake (P.R. of China). Italy, SIJLAB (2007)

8. Bi, J.J.: Introduction to Offshore Mechanics. Tongji University Press, Shanghai, China (1989) (in Chinese)

9. Hallam, M.G., Heaf, N.J., Wootton, L.R.: Dynamics of Marine Structures, report UR8, 2nd edn. CIRIA Underwater Engineering Group (1978)

10. Ge, F.: Dynamic response of submerged floating tunnel under wave and current conditions. [Ph.D. Dissertation], Institute of Mechanics, Graduate University of Chinese Academy of Sciences, Beijing, China (2006) (in Chinese)

11. Susumu, M., Akihide, T., Yuzo, M., Hiroshi, K., Toshihiko, Y., Hiroshi, S.: Experimental study on characteristics of submerged floating tunnels under regular waves. In: Proceeding 3rd Symposium on Strait Crossings, Alesund, pp. 667-674 (1994)

12. Long, X., Ge, F., Wang, L., Hong, Y.S.: Dynamic response of submerged floating tunnel with different buoyancy-weight ratios under wave and current loads. In: Proceedings of the Seventeenth National Conference on Structural Engineering (III), Wuhan, China, pp. 556-561 (2008) (in Chinese)

13. Kanie, S., Mikami, T.: Effect of non-linearity in restoring force on dynamic response of SFT. In: Proceeding 4th Symposium on Strait Crossings, Bergen, Norway, pp. 529-534 (2001) 